\title{
TRAJECTORY ERROR MINIMISATION METHOD DUE TO TRANSIENT REGIME VARIATION OF INDUSTRIAL ROBOTS KINEMATIC LINKS
}

\author{
ANDREI LUNCANU ${ }^{* 1}$, GHEORGHE STAN ${ }^{1}$ \\ I "Vasile Alecsandri" University of Bacau, Calea Marasesti 157, Bacau, 600115, Romania
}

\begin{abstract}
In the current industry, industrial robots are gaining more and more ground to classical positioning methods, especially due to the ratio of workspace / volume of the industrial robot. For this reason, methods of minimization of trajectory errors are necessary. Among the multitudes of factors that affect the trajectory precision is the difference between the programmed transient regime and the measured transient regime of the kinematic link used in the structure of the industrial robots. In this paper is presented the method of measurement the transient regimes of the end-effector and a method of compensation of the trajectory error.
\end{abstract}

Keywords: trajectory, industrial robots, kinematic links

\section{INTRODUCTION}

Through the research carried out on the industrial robot structure [1], only some of the positioning and trajectory errors produced, in particular by the dimensional and geometrical deviations of industrial robots, can be minimized. But another class of factors that influence the precision of industrial robots is kinematic type. By investigating kinematic factors that are influencing the trajectory tracking precision the industrial robot it has been determined that the variation of the transient regime of the kinematic links present in the kinematic structure of the industrial robots is a dominant factor. For this reason, it is necessary to implement a method of analysis and correction of the variations of the transient regime. The purpose of using this method is to obtain a transient regime as close as possible to the programmed transient regime. By applying the method presented in this article, an optimized transient regime is obtained and the trajectory tracking precision is improved. In order for this method to work, it is necessary to use the experimental stand presented in the article, in which all the kinematic links can control and the transient regime of the end-effector can be determined.

\section{DETERMINATION OF THE INFLUENCE PRODUCED BY THE TRANSITORY REGIME ON THE TRAIECTORY TRACKING PRECISION}

According to special studies, the transient regime is important in both the positioning and in trajectory generation of an industrial robot. For this reason, the industrial robot trajectory must be very well defined. But in many cases the trajectory required for a technological process is not the trajectory generated by the industrial robot. The main reasons why the trajectory is not following the intended track is due to the aging of mechanical elements within the industrial robot structure, a troublesome servomotor controller, or a dynamic factor due to the inadequate inertial moment calculation. By analyzing the structures of the industrial robots [2] it has been determined that most structures of the kinematic chains used by the robot designers adhere to the block diagram

\footnotetext{
*Corresponding author, email: andreiluncanu@yahoo.com
}

(c) 2019 Alma Mater Publishing House 
of the kinematic translation chain shown in Figure 1 and the block diagram of the kinematic rotation chain shown in Figure 2. By comparing block diagrams in Figure 1 and Figure 2 we find that the kinematic chain diagrams share these elements: CNA - analog numerical converter, D - driver, SM - servomotor, T - motion transmitting system, TV - speed transducer, TP - position transducer and S - Ball Screw.

The guides of the translational kinematic chain is a particular case since the position transducer is mounted on the output element of the transmission system - T.

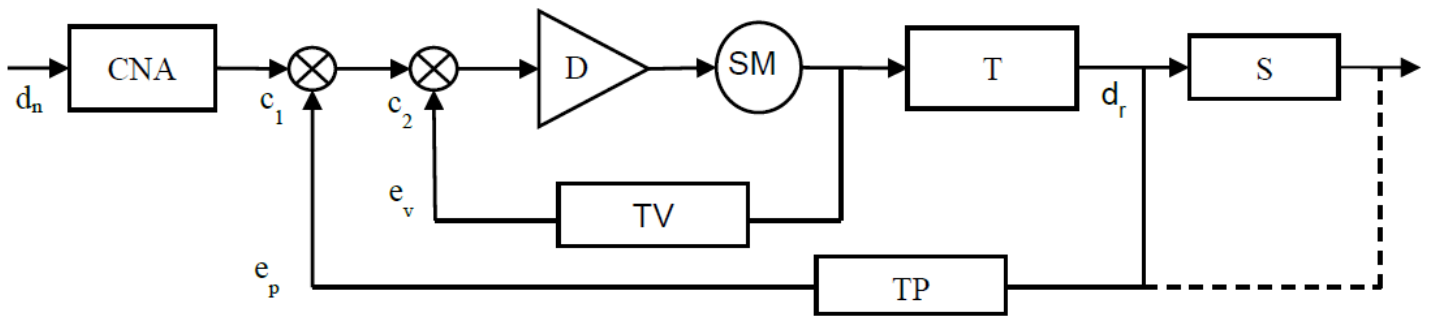

Fig. 1. Bloc diagram of the linear kinematic chain.

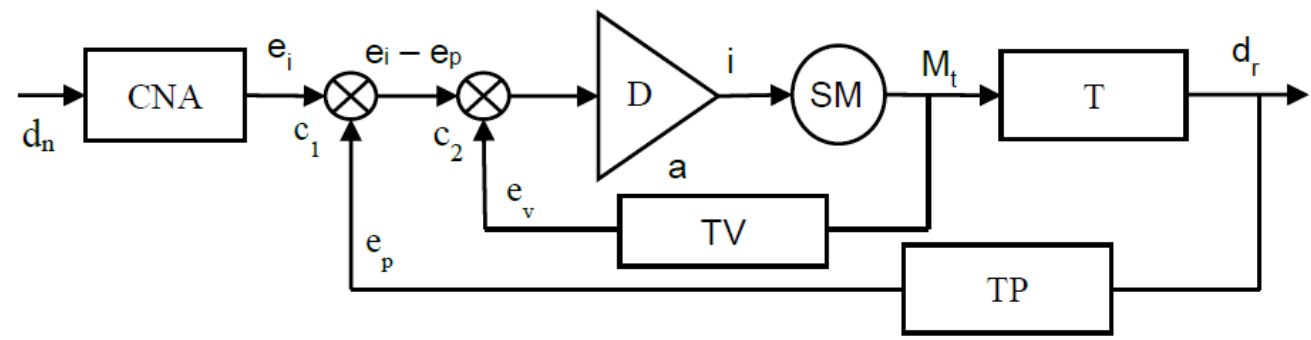

Fig. 2. Bloc diagram of the rotation kinematic chain.

To simplify the equation, we consider that actuator control does not influence the position control and the block diagram of the kinematic chain becomes the one shown in Figure 3.

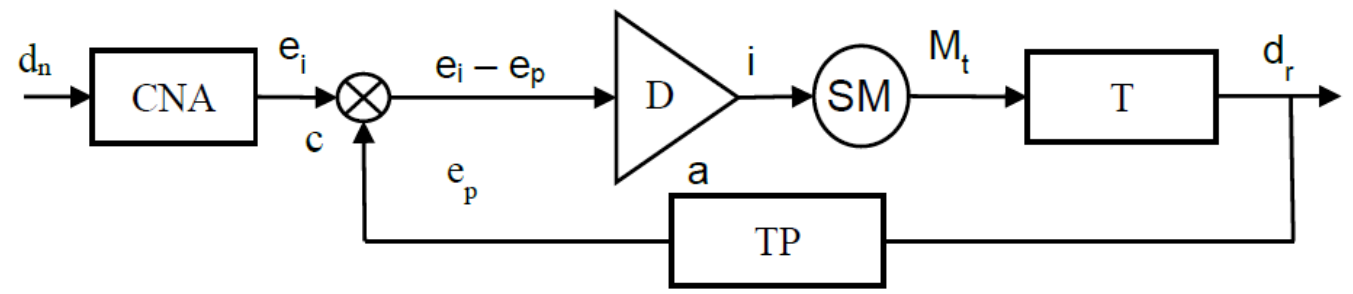

Fig. 3. Block diagram of the simplified kinematic chain.

In order to establish the final transfer function the substitutions of the block diagram must be made. $G_{I}$ is the transfer function of the analog numerical converter shown in equation (1). $G_{2}$ is the transfer function of driver shown in equation (2). $G_{3}$ is the transfer function of the servomotor shown in equation (3). $G_{4}$ is the transfer function of the transmission system shown in equation (4). $G_{5}$ is the transfer function of the position transducer shown in equation (5).

$$
\begin{aligned}
& G_{1}=\frac{e_{1}}{d_{n}} \\
& G_{2}=\frac{i}{e_{i}-e_{p}} \\
& G_{3}=\frac{M_{t}}{i} \\
& G_{4}=\frac{d_{r}}{M_{t}}
\end{aligned}
$$




$$
G_{5}=\frac{d_{r}}{e_{p}}
$$

By replacing, the transfer function blocks equations in the simplified block diagram of the kinematic chain, the resulted block diagram of the kinematic chain, shown in Figure 4, is obtained.

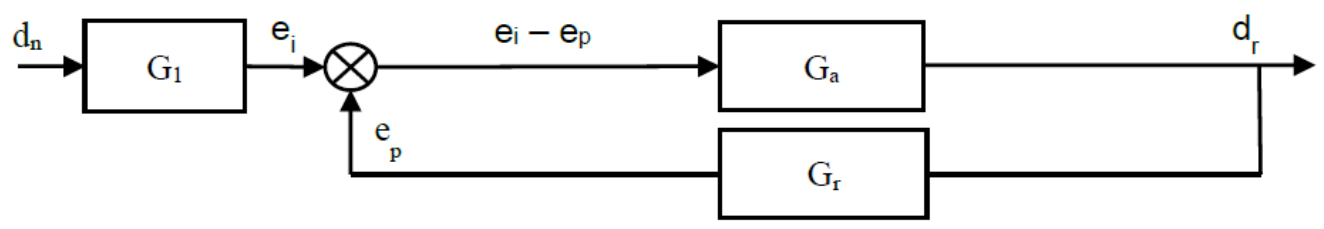

Fig. 4. The resulted block diagram of the kinematic chain.

In Figure $4 G_{a}$ is the transfer function resulting from calculation from the $G_{2}, G_{3}$ and $G_{4}$ transfer functions. The final transfer function of the kinematic chain of rotation is shown in equation (6).

$$
G_{S}=\frac{d_{r}}{d_{n}}=\frac{G_{1} \cdot G_{2} \cdot G_{3}}{s^{2} \cdot J+G_{1} \cdot G_{2} \cdot G_{3}}
$$

\section{EXPERIMENTAL DETERMINATION OF THE INFLUENCES PRODUCED BY THE DIFFERENCES BETWEEN THE REQUIRED TRANSIENT REGIME AND THE GENERATED TRANSIENT REGIME ON THE TRAJECTORY TRACKING PRECISION}

In order to determine the influences caused by the differences between the required and measured transient regime it is necessary to use the use of an experimental stand [3]. Figure 5 presents the experimental stand used to determine the influences produced by this phenomenon.

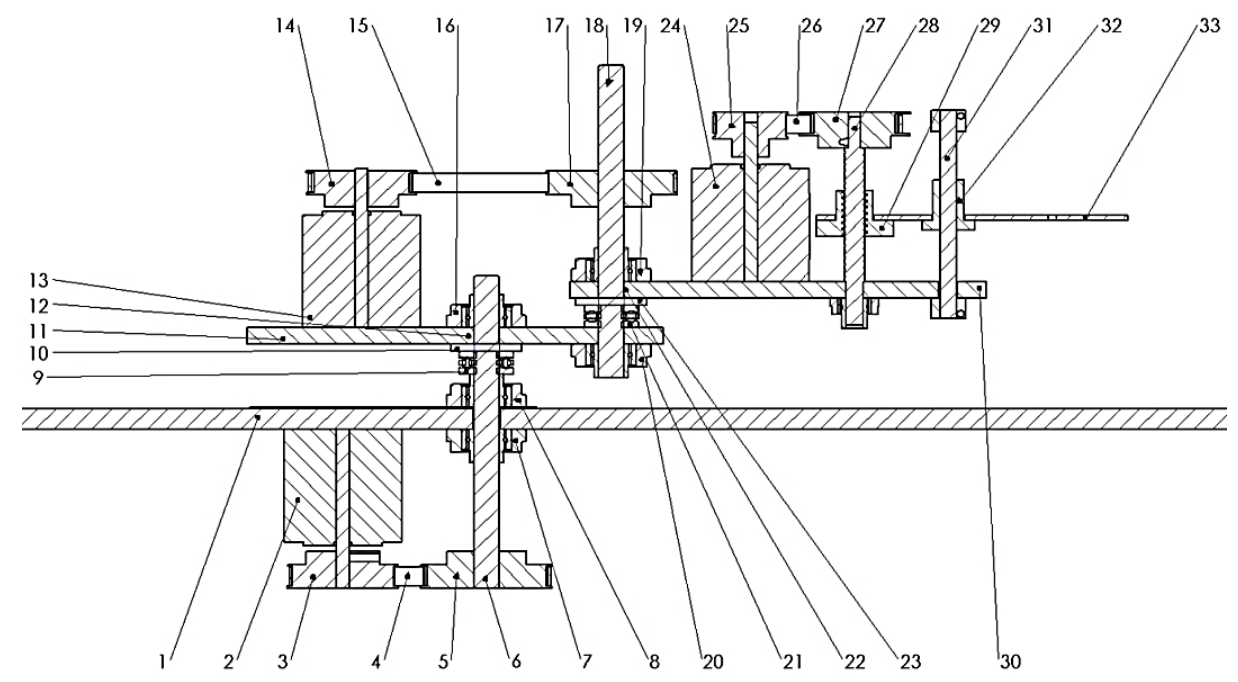

Fig. 5. Experimental stand used for determining the effects of transient regime deviations.

The kinematic links use the servomotors (2), (13) and (24) as actuators. The transmission of the motion from the servomotor (2) of the kinematic link $C_{l}$ is done throw the toothed timing belts transmission mechanism. The transmission mechanism consists of one 25-T5 toothed belt wheel (3), one 32-T5 toothed belt wheel (5) and one 300-T5 toothed belt (4). The 25-T5 toothed belt wheel (3) is mounted on the servomotor shaft (2), the 32-T5 toothed belt wheel (5) is mounted on the $\theta 12$ axle (6). The 300-T5 toothed belt (4) is mounted and pre-tensioned between the 25-T5 toothed belt wheel (3) and the 32-T5 toothed belt wheel (5). The $\theta 12$ axle (6) is mounted on the support structure (1) with the radial ball bearings (7) and (8). The $\theta 12$ axle (6) is mounted on the support structure of the kinematic link $C_{l}$ (11) with the radial ball bearing (16) and the axial ball bearing (9). The 
mechanical element (10) is used to introduce the reference position deviation of the kinematic link and the mechanical element (12) is used for inserting the radial and axial runout deviations. The transmission from the servomotor (13) to the kinematic link $C_{2}$ is done throw the toothed timing belt transmission mechanism. The transmission mechanism consists of one 25-T5 toothed belt wheel (14), one 32-T5 toothed belt wheel (17) and one 400-T5 toothed timing belt (15). The 25-T5 toothed belt wheel (14) is mounted on the servomotor shaft (13), the 32-T5 toothed belt wheel (17) is mounted on the $\theta 12$ axle (19) and the toothed timing belt (15) is mounted and pre-tensioned between the 25-T5 toothed belt wheel (14) and the 32-T5 toothed belt wheel. The axle $\theta 12$ (18) is mounted between support structure of the kinematic link $C_{I}$ (11) and the support structure of the kinematic link C2 (26) as a sandwich type assembly with the radial ball bearings (19) and (20) and the axial ball bearing (22). The mechanical element (23) is used to introduce the reference position deviation of the kinematic link and the mechanical element (24) is used for inserting the radial and axial runout deviations. The transmission of the movement from the servomotor (24) and the end-effector (33) is obtained by using a toothed belt transmission system and a screw-nut transmission system. The toothed belt transmission consists of one 24T5 toothed belt wheel (25), 25-T5 toothed belt wheel (27) and one 225T5 toothed timing belt (26). The screwnut transmission mechanism consists of the $12 \times 3$ trapezoidal-threaded screw (28) and the trapezoidal threaded flange nut (29). The clamping element is mounted on the end-effector support structure (33). The Renishaw ML10 Gold linear measurement system shown in Figure 6 is used to measure the influence of transient regime deviations.

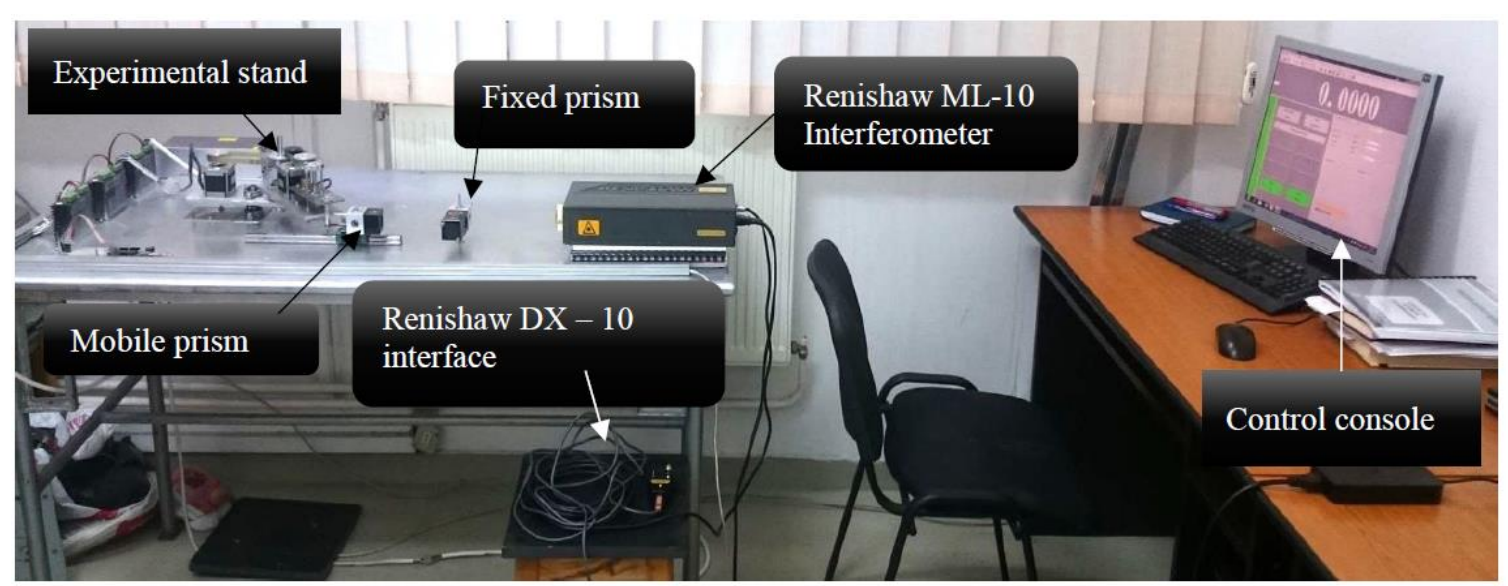

Fig. 6. Liniar trajectory measurement with Renishaw ML10 gold interferometer.

To determine the errors caused by the deviations between the programmed transient regime and the measured transient regime, it is necessary to use a predefined trajectory. Since the experimental stand uses 3 kinematic couplings, it is necessary to use three synchronized transient regimes. The trajectory used to apply the measurement method is shown in Figure 7.

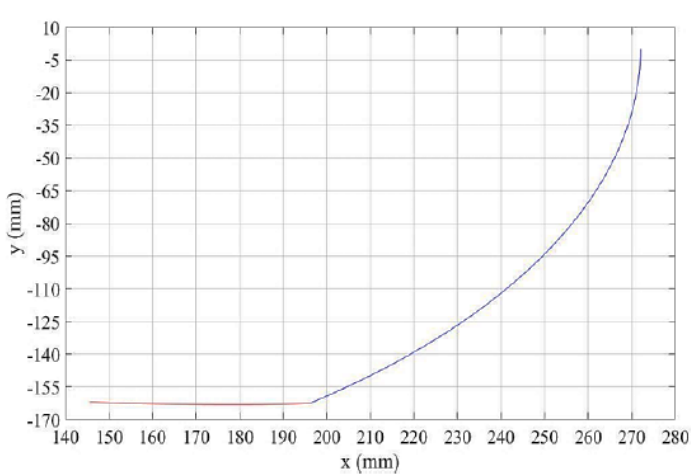

a.

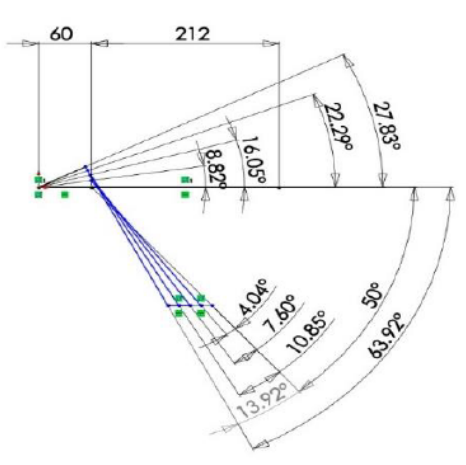

b.

Fig. 7. Trajectory programmed to apply trajectory: a. Programmed trajectory b. Angles required to implement the trajectory. 
In Figure 7.a. the programmed trajectory consists of a position trajectory from the point 0 of the experimental stand to the measurement starting point symbolized with a blue line and a linear trajectory used for measurement with the Renishaw ML-10 Gold interferometer symbolized with a red line. Figure 7.b. shows the method of determining the trajectory based on the dimensional factors of the experimental stand.

\section{REZULTS AND CONCLUSIONS ON THE TRAJECTORY ERROR MINIMISATION THROW TRANSIENT REGIME OPTIMISATION}

By applying the measurement method [4] to the linear trajectory of the experimental booth, the positioning graph of the end-effector, shown in Figure 8, is obtained.

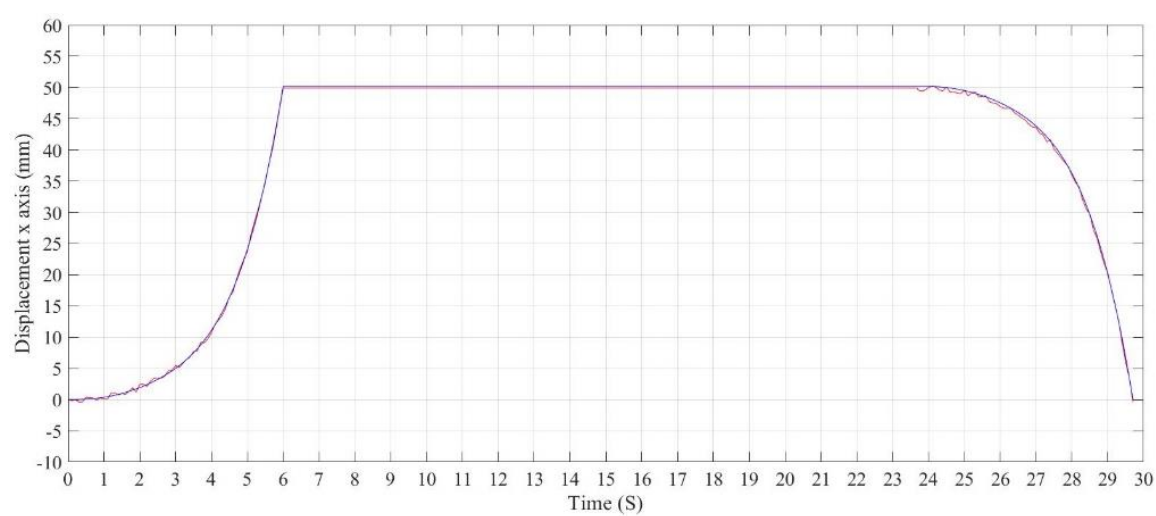

Fig. 8. Generated and programmed transient regime before optimization.

In Figure 8, the programmed transient regime is symbolized with a blue line, and the transient regime generated by the end-effector is symbolized with a red line. By applying a comparison method to the transient regimens, the trajectory error graph produced by differences between the generated and programmed transient regime is shown in Figure 9.

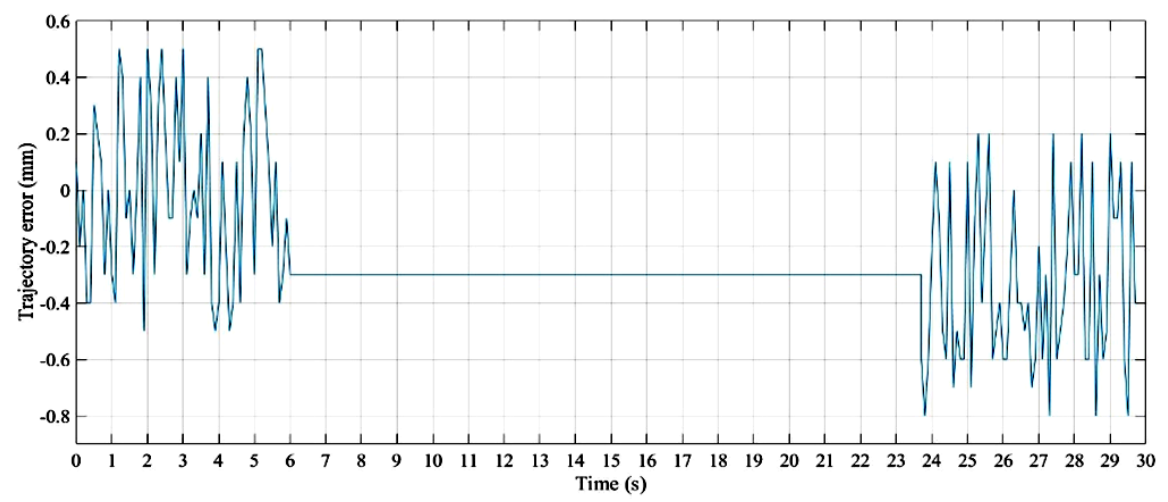

Fig. 9. Trajectory tracking error obtain before optimization.

As can be seen from Figure 9, the maximum trajectory error is $0.5 \mathrm{~mm}$ for acceleration and $0.8 \mathrm{~mm}$ for deceleration. By analyzing the precision standards of industrial robots in the current industry, it was determined that the trajectory error generated by the experimental stand was three times higher than that of an industrial robot.

In practice all the kinematic couplers of an industrial robot that are using a servomotor as the main actuator have a PID regulator in their command structure. After using the method of PID optimizing of the servomotors a new series of measurements using the Renishaw ML-10 laser measurement system have been made. As a result the transient regimen obtained after the PID optimization is shown in Figure 10. 
As can be seen from Figure 10, the transient regime generated by the end-effector is almost the same as the programmed transient regime. By using the comparison system of the transient regime generated by the endeffector and programmed transient regime, the obtained trajectory error is shown in Figure 11.

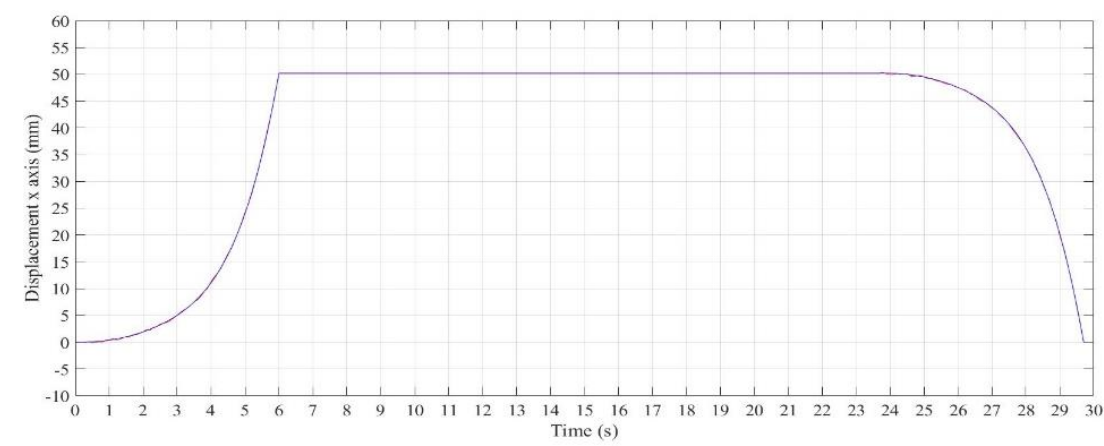

Fig. 10. Generated and programmed transient regime after optimization.

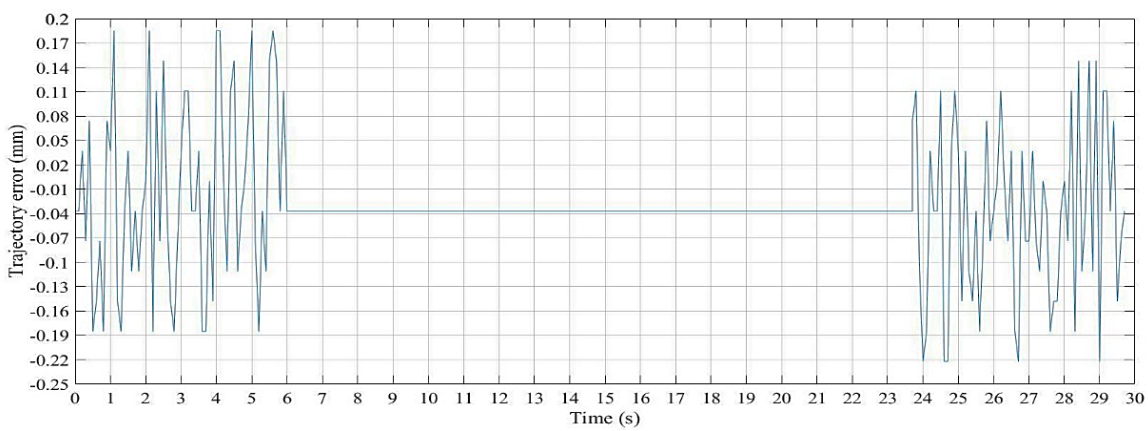

Fig. 11 Trajectory tracking error after optimization.

As can be seen from the resulting trajectory error graph, the maximum error of $0.18 \mathrm{~mm}$ at acceleration and $0.22 \mathrm{~mm}$ at deceleration, an improvement of approximately 3 times the trajectory error.

In conclusion, by using this method of optimization of the transient regime it is allowed to minimize the trajectory error of the end-effector mounted on an industrial robot. Since most industrial robots use a PID regulator in the control structure, this method can be used on both existing control structures and in future control structures.

\section{REFERENCES}

[1] Das, M.T., Dulger, L.C., Mathematical modelling, simulation and experimental verification of a SCARA robot, Simulation Modelling Practice and Theory, vol. 13, 2005, p. 257-271.

[2] Siciliano, B., Sciavicco, L., Villani, G., Robotics: Modelling, Planning and Control, Springer, 2009.

[3] Tarun, P., Singh, S.P., Chandan, S., Forward and inverse kinematic analysis of robotic manipulators, International Research Journal of Engineering and Technology, vol. 4, 2017.

[4] Zlajpah, L., Simulation in robotics, Mathematics and Computers in Simulation, 2008. 